\title{
Value of Apparent Diffusion Coefficient and Relative Cerebral Blood Volume Calculated by MRI for Grading of Brain Tumors
}

\author{
ISMAIL M. ABDEL HAKIM, M.Sc. and AHMED TOHAMY AHMED, M.D. \\ The Department of Diagnostic Radiology, Faculty of Medicine, Suez Canal University, Egypt
}

\begin{abstract}
Background: MRI is the standard technique for diagnosis of brain tumors, but the challenge is determination of brain tumor grade.

Aim of Study: To assess the role of Apparent Diffusion Coefficient (ADC) and relative Cerebral Blood Volume (rCBV) in brain tumors grading.

Patients and Methods: Our prospective comparative analytic study involved 26 patients that were diagnosed to have brain tumors. All patients were subjected to revision of their medical history, full neurological and cognitive functions assessment, anatomical brain assessment by conventional MRI examination of the brain, diffusion and perfusion MRI with measurement of mADC \& rCBV values respectively. Correlation of results with the histopathological findings of the resected tumors as a gold standard were performed.

Results: The diagnostic capability of mADC by using cut off value of $<1.28 \times 10^{-3} \mathrm{~mm} / \mathrm{s}$ had a sensitivity of $81.2 \%$, specificity of $100 \%$ at $p$-value of 0.0001 . All the low-grade tumors had low perfusion (rCBV $<3.22 \&<2.33$ ) while most of the high-grade tumors had high perfusion $(\mathrm{rCBV}>3.22 \&$ $>2.33)$ with significant difference in between $(p=0.001 \&$ 0.002 ). The diagnostic capability of rCBV by using cut off value of $>3.22 \&>2.33$, had a sensitivity of $68.4 \%, 90.9$ respectively and a specificity of $100 \%$ at $p$-value of $<0.00$. Multivariant logistic regression revealed that $\mathrm{rCBV}$ higher than 2.33 and mADC !9 1281.34 fitted the statistical regression for prediction of high grade tumors in intra-axial brain tumors $(p=0.002)$

Conclusion: Diffusion and perfusion imaging are useful for characterizing intra-axial brain tumors. rCBV measurement can be used to determine the grade of glioma with no added advantage of ADC map.
\end{abstract}

Key Words: Diffusion - Perfusion - MRI - Brain tumor Glioma.

\section{Introduction}

BRAIN tumors are the second leading cause of cancer-related deaths in children under age of 20

Correspondence to: Dr. Ismail M. Abdel Hakim, The Department of Diagnostic Radiology,

Faculty of Medicine, Suez Canal University, Egypt and the second leading cause of cancer-related deaths in males ages 20-39 in the United States $[1,2]$.

MRI is the standard technique for brain tumor diagnosis. It is used in combination with other imaging modalities, such as Computed Tomography (CT), and Positron Emission Tomography (PET) to provide the most exact information about tumor morphology and metabolism [3]. The most frequent challenge in the diagnosis of brain tumor is the differential diagnosis of a solitary intra-axial brain tumor. In such cases, gross tumor resection or stereotactic biopsy is required to reach a definitive diagnosis [4]. Qualitative interpretation of basic brain tumor MRI (including T2-weighted images and gadolinium [Gd]-enhanced T1-weighted images) remains the backbone of brain tumor imaging in a significant number of cases [5]. However, these techniques fail to allow confident and correct differential diagnosis, grading, and monitoring of the brain tumors.

Nowadays the role of neuroimaging in patients with brain tumors is no longer simply to evaluate structural abnormality, it allows characterization of morphologic as well as biologic alterations to diagnose and grade brain tumors as well as to monitor and assess treatment response and patient prognosis [6]. The grade of brain tumor is pivotal in the treatment decision, follow-up to monitor disease progression and therapeutic response. DWI \& perfusion weighted imaging play an important role in the transition of clinical MR imaging to one that combines structure with function. Perfusion MRI provides precise determination of the regional vascularity and degree of vascular proliferation unlike contrast enhancement that indicates disruption of the blood-brain barrier, which is an important parameter for the histopathologic grading of gliomas [7]. 
Thus the purpose of our study was to assess the role of Apparent Diffusion Coefficient (ADC) and relative Cerebral Blood Volume (rCBV) in brain tumors grading.

\section{Patients and Methods}

This is a analytic comparative prospective single centre study approved by the local institutional ethics committee; written informed consent was obtained from all patients.

\section{Study population:}

Our study consisted of a series of 26 patients (10 male patients and 16 female patients with age range from 11-79 years) were diagnosed as to have brain tumors who underwent to pre-operative brain MRI over a period of 1 year from January 2017 to January 2018. Inclusion criteria involved patients with brain tumors (based on symptoms, clinical examination, conventional MRI \& histopathological findings), while the exclusion criteria involved absolute contraindications to MR imaging (implanted defibrillator or pacemaker, cochlear implant, some types of clips used on brain aneurysms), and patients refusing to contribute in the study.

All patients were asked about their medical history, past illness and neurological problems, and subjected to full neurological and cognitive function assessment.

Anatomical brain assessment by using conventional MRI assessment (T $1 \mathrm{~W}, \mathrm{~T} 2 \mathrm{~W}$, FLAIR \& T1 post contrast sequences), diffusion \& perfusion were done for all patients selected according to the inclusion and exclusion criteria. mADC \& rCBV values were measured and correlated with the pathology results of the masses which were obtained post-operatively.

\section{Magnetic resonance imaging protocol:}

All patients underwent a 1.5 Tesla MR of the brain by using MR scanner (Philips Medical Systems, Achieva) and post processing was done by Philips work station using software (Extended work space, version 2.6).

\section{Patient's preparation:}

The ferromagnetic materials were taken off. The procedure was explained to the patient, and then the patient was asked to lie supine and instructed not to move during the study.

\section{MRI protocol:}

Anatomical brain assessment by using conventional MRI assessment (T $1 \mathrm{~W}, \mathrm{~T} 2 \mathrm{~W}$, FLAIR \& T1 post contrast sequences), diffusion and perfusion- weighted MR images were obtained. DiffusionWeighted (DW) imaging comprised an echoplanar spin-echo sequence with the following parameters: TR 4247, TE 95, EPI factor 77, Field of View (FOV) $230 \mathrm{~mm}$, slice thickness $5 \mathrm{~mm}$, slice gap $1 \mathrm{~mm}$, number of excitations 1 , matrix 77 X 256 , number of slices 22 , acquisition time 30 s. The DW images were acquired at $b$-values of 0 and 1000 $\mathrm{s} / \mathrm{mm}^{2}$. An isotropic image was constructed in real time, pixel by pixel, as an average of the signal intensities in three orthogonal directions. ADC maps were generated from the DW images. Dynamic susceptibility contrast-enhanced MRI (DSCMRI) was performed to acquire perfusion images. An echoplanar gradient-echo sequence with the following parameters was employed: TR 17, TE 8, flip angle 7, EPI factor 7, FOV 220mm, slice thickness $3.5 \mathrm{~mm}$, slice gap $0 \mathrm{~mm}$, number of excitations 1 , matrix $64 \times 64$, number of slices 60 series of 22 slices, acquisition time $77 \mathrm{~s}$. This technique is highly sensitive to $\mathrm{T} 2 *$ changes due to its very long TE. Image acquisition began simultaneously with contrast agent injection. A standard dose $(0.2 \mathrm{ml} / \mathrm{kg}, 0.1 \mathrm{mmol} / \mathrm{kg})$ of Gadobenate Dimeglumine was injected using an automated power injector at a flow rate of $6 \mathrm{ml} / \mathrm{s}$ followed by a $40-\mathrm{ml}$ saline flush at the same rate.

\section{MRI image analysis:}

All MR images were reviewed by consensus of two experienced radiologists blinded to the clinical information \& final histopathological results. Images were transferred to an offline workstation for post processing.

For rCBV calculation; Regions of Interest (ROI) were manually placed as two circles; the first around the tumor and the second around normal contralateral brain tissue on the derived rCBV maps, the CBV was then calculated for each, the same process was repeated 3 times in different areas of the brain tumor. The highest CBV value of each tumor was divided by the CBV value of the contralateral normal brain tissue to provide the rCBV index values used in this study.

For ADC calculation; Regions of Interests (ROI) were manually placed as 3 separate ellipses where the tumor was, then the ADC was calculated for each and the mean ADC (mADC) was obtained.

\section{Results}

The study involved 10 male patients (37\%) and 16 female patients (63\%), age of patients ranged from 11-79 years, the age range group of 30-39 represented $(22.2 \%)$. Based on histopathological results; metastasis represented the majority of cases 
$(22.2 \%)$ and the lowest number of cases was vestibular schwannoma $(3.7 \%)$, other pathological entities involved medulloblastoma, glioblastoma multiform, meningioms, astocytoma, anaplastic astrocytoma, central neurocytoma, gliosarcoma, anaplastic oligidendroglioma Figs. $(1,2)$.
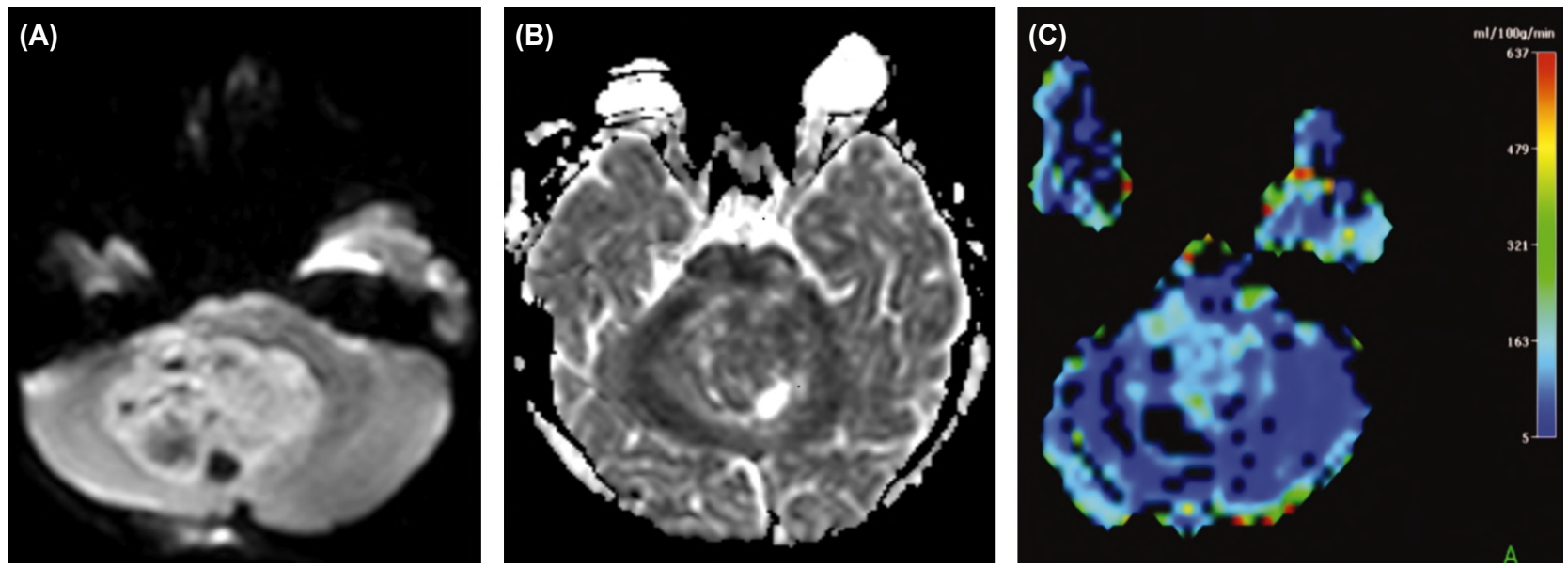

Fig. (1): MRI brain of a 11-year-old boy with headache showing a 4 th ventricular mass pathologically proven to be medulloblastoma. (A) Diffusion Weighted Image 'DWI', (B) Apparent Diffusion Coefficient 'ADC'. The mass appeared to be hyperintense on DWI that turns to be slightly dark on ADC map denoting diffusion abnormality. Mean ADC $=0.952$ X $10 \mathrm{~mm} / \mathrm{s}$ (c) Accompanying rCBV color map shows increased perfusion within the mass. Lesion shows CBV values in the lower range. The maximum $\mathrm{rCBV}=2.04$.
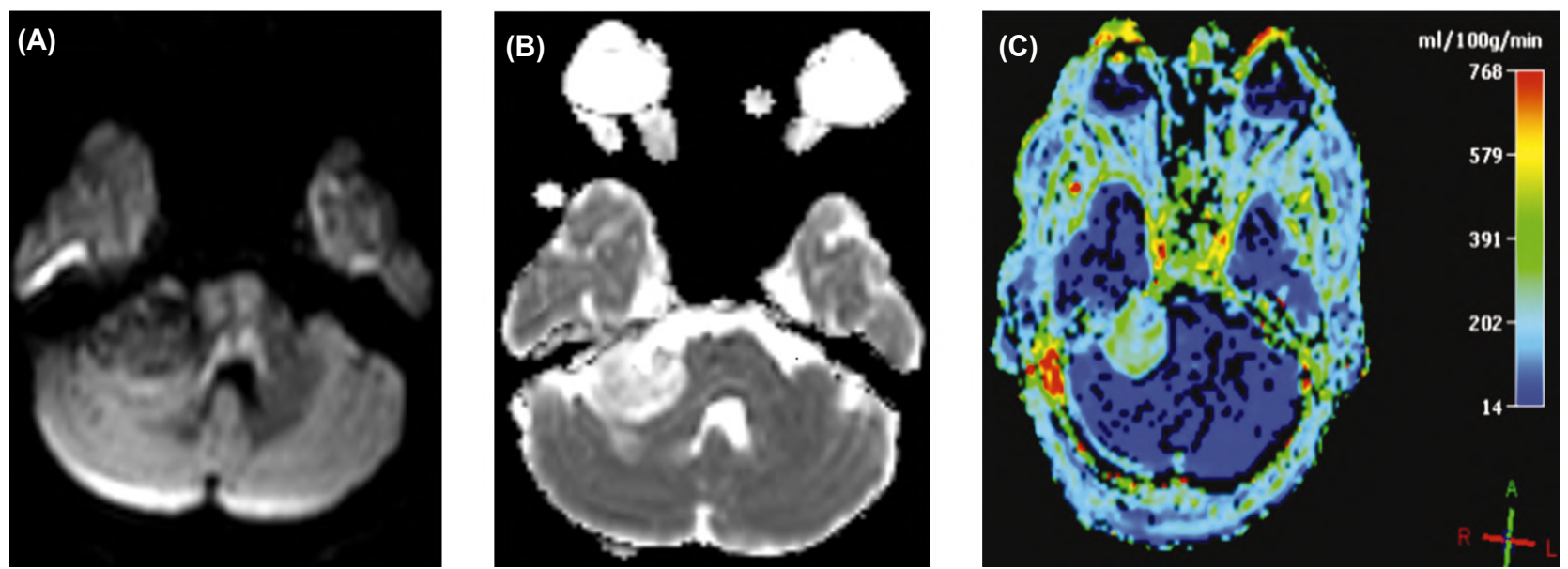

Fig. (2): MRI brain of a 64-year-old female with headaches showing a Rt. CPA mass pathologically proven to be acoustic neuroma. (A) Diffusion Weighted Image 'DWI', (B) Apparent Diffusion Coefficient 'ADC'. The mass appearęd to be hypointense on DWI with correspondingly bright ADC map denoting no diffusion restriction. Mean ADC $=1.507 \times 10^{-5 m} \mathrm{~mm} / \mathrm{s}$ (c) Accompanying rCBV color map shows increased perfusion within the mass. Lesion shows CBV values in the lower range. The maximum $\mathrm{rCBV}=3.22$.

The diffusion pattern of intra-axial brain tumors compared to their WHO grade revealed that all low-grade tumors had mADC higher than the cutoff point, while most of the high-grade tumors had mADC lower than the cutoff point with a significant difference; $p$-value $=0.0187$. Receiver Operating Curve analysis (ROC) for assessment of mADC revealed that Area Under Curve (AUC) was 0.875 (at 95\% confidence interval 0.636-0.981). The results of diagnostic capability of mADC by using cut off value of $\leq 1.28 \times 10^{-3} \mathrm{~mm}^{2} / \mathrm{s}$ had a sensitivity of $81.2 \%$, specificity of $100 \%, p$-value of 0.0001 Fig. (3).

All low-grade tumors had low perfusion (rCBV $<3.22$ ), while most of the high-grade tumors had high perfusion ( $\mathrm{rCBV}>3.22$ ) with significant difference; $p$-value $=0.0014$ (Table 1$)$. ROC curves for assessment of rCBV revealed that (AUC, 0.829; at $95 \%$ confidence interval $0.636-0.945)$. The results of diagnostic capability of rCBV by using cut off value of $>3.22$ had a sensitivity of $68.4 \%$, specificity of $100 \%, p$-value of $<0.000$ Fig. (4). 


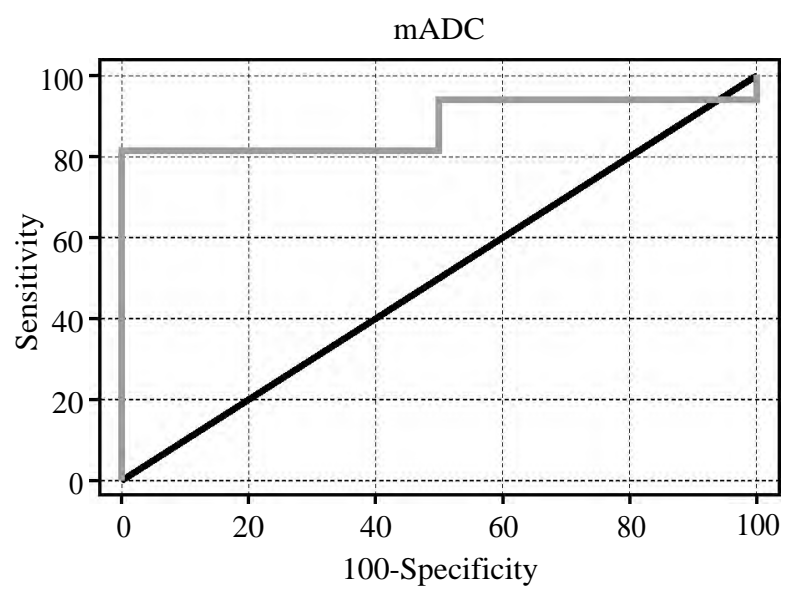

Fig. (3): ROC curve for $\mathrm{mADC}$ shows that the $\mathrm{m}_{2} \mathrm{ADC}$ by using cut off value of $\leftrightarrow 1.28 \times 10 \mathrm{~mm} / \mathrm{s}$ had a sensitivity of $81.2 \%$ and a specificity of $100 \%$ at a $p$-value of 0.0001 .

Table (1): The perfusion pattern of all brain tumors (intra + extra) compared to their WHO grade.

\begin{tabular}{llllll}
\hline WHO & \multicolumn{3}{c}{ Perfusion-3.22 } & $\begin{array}{c}\text { Chi- } \\
\text { Square }\end{array}$ & $\begin{array}{c}p- \\
\text { value }\end{array}$ \\
\cline { 2 - 5 } & Low "0" & High "1" & & \\
\hline Low "0" & $8(100.0 \%)$ & $0(0.0 \%)$ & $8(29.6 \%)$ & 10.165 & $p=$ \\
High "1" & $6(31.6 \%)$ & $13(68.4 \%)$ & $19(70.4 \%)$ & & $0.0014 *$ \\
\hline & $14(51.9 \%)$ & $13(48.1 \%)$ & $27(100.0 \%)$ & & \\
\hline
\end{tabular}

*: Statistically significant difference.

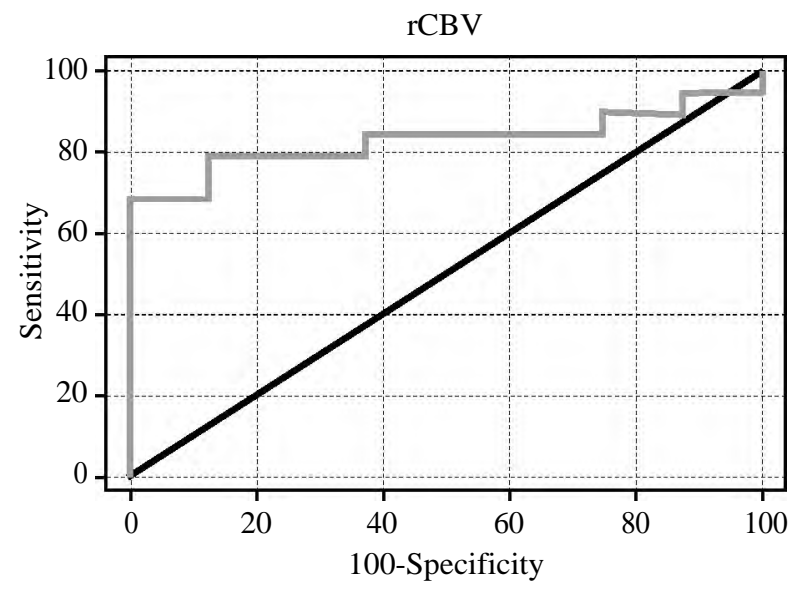

Fig. (4): ROC curve for rCBV shows that the rCBV by using cut off value of $>3.22$ had a sensitivity of $68.4 \%$ and a specificity of $100 \%$ at a $p$-value of $<0.0001$.

Table (2): The perfusion pattern of brain gliomas compared to their WHO grade.

\begin{tabular}{|c|c|c|c|c|c|}
\hline \multirow{2}{*}{$\begin{array}{l}\text { WHO } \\
\text { grade }\end{array}$} & \multicolumn{3}{|c|}{ Perfusion $>2.33$} & \multirow{2}{*}{$\begin{array}{l}\text { Chi- } \\
\text { Square }\end{array}$} & \multirow{2}{*}{$\begin{array}{c}p- \\
\text { value }\end{array}$} \\
\hline & Low "0" & High "1" & & & \\
\hline Low "0" & 3 & 0 & $3(21.4 \%)$ & 8.864 & \\
\hline \multirow[t]{2}{*}{ High "1" } & 1 & 10 & $11(78.6 \%)$ & & $0.0029 *$ \\
\hline & $4(28.6 \%)$ & $10(71.4 \%)$ & $14(100.0 \%)$ & & \\
\hline
\end{tabular}

*: Statistically significant difference.
All low-grade gliomas showed low perfusion $(\mathrm{rCBV}<2.33)$ while most of the high-grade gliomas showed high perfusion $(\mathrm{rCBV}>2.33$ ) with significant difference $(p=0.0029)$. It had an ODDS Ratio (OR) of 49, $p$-value $=0.026$ (Table 2). Meanwhile ROC assessment of rCBV revealed that (AUC, 0.939 ; at $95 \%$ confidence interval 0.676-0.999). The results of diagnostic capability of rCBV by using cut off value of $>2.33$ had a sensitivity of $90.9 \%$, specificity of $100 \%, p$-value of $<0.0001$. There was a statistically significant difference between the perfusion in low-grade and high-grade Gliomas Fig. (5).

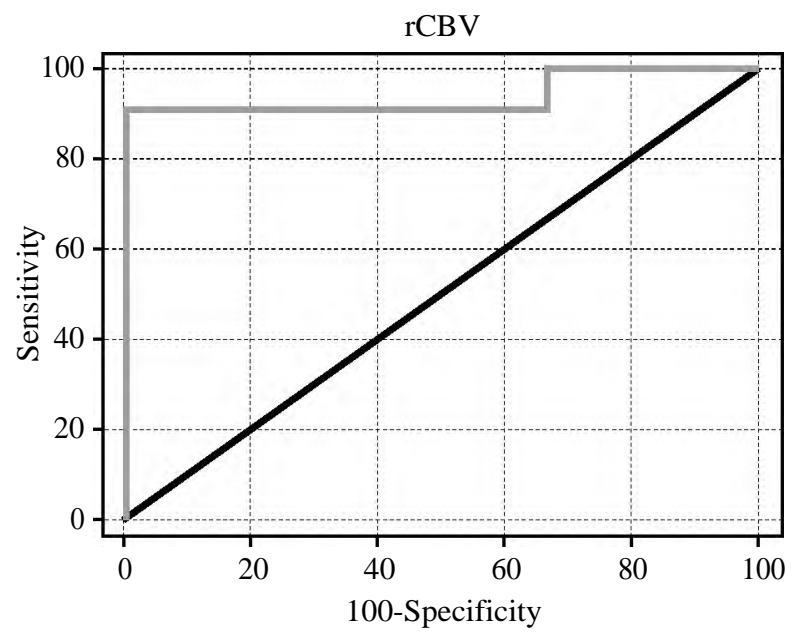

Fig. (5): ROC curve for $\mathrm{rCBV}$ shows that the rCBV by using cut off value of $>2.33$ had a sensitivity of $90.9 \%$ and a specificity of $100 \%$ at a $p$-value of $<0.0001$.

With multivariant logistic regression; an $\mathrm{rCB}_{2}$ higher than 2.33 and $\mathrm{mADC} \leq 1.28 \times 10^{-3} \mathrm{~mm}^{2} / \mathrm{s}$ fitted the statistical regression for prediction of high grade tumors in intra-axial brain tumors ( $p=$ 0.002 ).

\section{Discussion}

Intra-axial brain masses are a significant health problem. The major diagnostic challenge is to reliably, noninvasively, and promptly differentiate intra-axial tumors to avoid biopsy and imaging follow-up. Integration of diagnostic information from advanced MR imaging techniques can further improve the classification accuracy of conventional anatomic imaging. There is no universally accepted single threshold for rCBV that can help discriminate among the various intra-axial lesions [3].

Our study showed that the DWI can significantly differentiate intra-axial tumors into low and high grades according to WHO classification with a sensitivity and specificity of $81.2 \%$ and $100 \%$ respectively at a cutoff point of $\leq 1.28 \times 10^{-3} \mathrm{~mm}^{2} / \mathrm{s}$. 
In agreement with our results; Kono et al., [8] reported a statistically significant difference of ADC values between glioblastomas and grade II astrocytomas. An inverse relationship between diffusion and tumor cellularity was observed and lower ADC values suggested malignant gliomas, higher ADC values suggested low grade astrocytomas. Fan et al., [9] showed that DWI is useful in the differentiation of non-enhancing gliomas, as $A D C$ values in anaplastic astrocytomas were significantly lower in the solid portions of the tumors compared to low grade glioma. Yang et al., [10] agree with other authors. This finding may be explained by tortuousity of the interstitial space and the resultant limitation in water movement [11].

On the other hand, Rizzo et al., [12] concluded that DWI are inadequate to provide information about the degree of differentiation of glial tumors. Lam et al., [13] failed to find a significant difference between the two grades of tumor.

Current basic biology research focuses on the interactive role of tumor hypoxia, gene expression, and macrophage activation, in the transition from normal permeability and blood volume, to increased permeability and volume, and finally to frank neoangiogenesis, during transformation from lowgrade glioma to GBM. In these neocapillaries, deficiency or absence of basal lamina and pericytes and reduced endothelial expression of occludins and other cell surface proteins result in large endothelial gaps or fenestrations and leaky intercellular tight junctions that together produce the markedly increased capillary permeability [14].

Our results highligthed the role of perfusion in differentiation of lower and higher grades of tumors according to WHO standard with statistically significant results. All the low-grade gliomas showed low perfusion ( $\mathrm{rCBV}<2.33$ ), while most of the high-grade gliomas showed high perfusion (rCBV $>2.33$ ) with statistically significant difference in between both types ( $p$-value $=0.0029)$.

The results of a study by Ellika et al., [15] on 14 patients of gliomas revealed that the mean rCBV in the high-and low-grade gliomas was $3.06 \pm 1.35$ and $1.44 \pm 0.42$, respectively, with a statistically significant difference between the 2 groups ( $p=$ $.005)$. Cut points of $>1.92$ for $\mathrm{rCBV}$ ( $85.7 \%$ sensitivity and $100 \%$ specificity) were found to identify the high-grade gliomas. Shin et al., [16] used a threshold of 2.93 , with a sensitivity of $90.9 \%$ and a specificity of $83.3 \%$. Hakyemez et al., [17] used a threshold of 2.00 to differentiate low-and high- grade gliomas, with $100 \%$ sensitivity and $90.9 \%$ specificity. Lev and Rosen [18] described a threshold of 1.5 in discriminating between patients with low and high-grade gliomas, with a sensitivity and specificity of $100 \%$ and $69 \%$, respectively. Law et al., [19] showed a sensitivity and specificity of $95.0 \%$ and $57.5 \%$, respectively, by using 1.75 as the threshold value.

The results of our study revealed that the rCVB at the cutoff point of $>3,22 \&$ the ADC at the cutoff point of $\leq 1.28 \times 10^{-3} \mathrm{~mm}^{2} / \mathrm{s}$ can significantly distinguish low grade from high grade tumors according to WHO grading.

In agreement with our study, Rollin et al., [20] found that rCBV values were greater in high-grade than in low-grade tumors $(3.87 \pm 1.94$ versus $1.30 \pm$ 0.42 ). The differentiation between high-and lowgrade gliomas was not possible using diffusionweighted images and ADC values alone.

Other studies reported no significant role for diffusion and found a helpful role of perfusion, as in Hakyemez et al., [17], they found that dural metastasis and meningiomas could not be differentiated by qualitative assessment of conventional and diffusion MRI. The rCBV ratios for dural metastasis and meningiomas were $4.13 \pm 2.32$ and $7.32 \pm 4.10$ respectively with significant $p$-value $(=0.003)$. A study by Rizzo et al., evaluated DWI and PWI imaging in the characterization of cerebral tumors. Ninety-eight patients with cerebral tumors revealed that ${ }_{2} \mathrm{ADC}$ value of solid tumors $\left(0.64-3.5 \times 10^{-3} \mathrm{~mm} / \mathrm{s}\right)$. The rCBV value was 1.4 $(\sigma 0.66)$ in low-grade gliomas; $1.22(\sigma 0.25)$ in lymphomas; 4.5 ( $\sigma 0.85)$ in grade III gliomas [12]

Our results were comparable with the findings of Lev et al., [18] who reported $100 \%$ sensitivity and $69 \%$ specificity at a cutoff value of 1.5 for rCBV. Shin et al., [16], who found a cutoff value of 2.9 with $91 \%$ sensitivity and $83 \%$ specificity. Although the sensitivity was similar, our specificity was higher than the others, which could be contributed to higher rCBV cutoff value (3.22) in our study, to eliminate confounding and to minimize type 2 error (number of false-positives).

This study had some limitations, firstly; that we found no difference between the ADC values of low and high-grade gliomas, and this finding may have resulted from the small sample size of our study, so further more studies with large sample size are necessary to cover different WHO grades of tumors to confirm these findings. Secondly; manual ROI placement represented one of the 
technical challenges in our study because of possible sampling error of voxel selection specially in multicenteric tumors. Automated lesion segmentation could have been more accurate and reproducible.

\section{In Conclusion:}

Diffusion and perfusion imaging are useful for characterizing intra-axial brain tumors, in conjunction with conventional MR imaging. rCBV measurements can be used to determine the grade of glioma. We found no clear advantage of ADC map for the grading of gliomas. We recommended to include diffusion and perfusion imaging as part of the routine evaluation of brain tumors. A practical diagnostic algorithm that integrates advanced MR imaging features to help the practicing radiologist to differentiate patients with high grade glioma from those with low grade glioma so that biopsy can be dispensible.

\section{References}

1- Brain Tumor Statistics: Available via http://www.abta.org/ brain-tumor-information/diagnosis/malignant-benignbrain-tumors.html, 2017 [accessed January 2018].

2- Central Brain Tumor Registry Of The United States Fact Sheet: Available via http://www.cbtrus.org/factsheet/ factsheet.html, 2016 [accessed January 2018].

3- STEFAN B., ROLAND W., LUTZ-P.N., et al.: A survey of MRI-based medical image analysis for brain tumor studies. Phys. Med. Biol., 58: R97-R129, 2013.

4- MUSTAFA K., ÖZLEM Y., ELIF O., et al.: Rare and challenging extra-axial brain lesions: $\mathrm{CT}$ and MRI findings with clinico-radiological differential diagnosis and pathological correlation. Diagn. Interv. Radiol., 20 (5): 44852, 2014.

5- JAYASHREE K., ELIZABETH R., KYRRE E., et al.: Advanced Magnetic Resonance Imaging of the Physical Processes in Human Glioblastoma. Physics in Cancer Research, doi: 10.1158/0008-5472.CAN-14-0383, 2014.

6- JO C., NADIA W., JENS H., et al.: The role of positron emission tomography-computed tomography and magnetic resonance imaging in diagnosis and follow-up of multip lemyeloma. Haematologica, 99: 629-37, 2014.

7- BRUZZONE M.G., D'INCERTI L., FARINA L.L., et al.: The Quarterly Journal of Nuclear Medicine and Molecular Imaging: Official Publication of the Italian Association of Nuclear Medicine (AIMN) [and] the International Association of Radiopharmacology (IAR), 56 (2): 112 37, 2012.
8- KONO K., INOUE Y., NAKAYAMA K., et al.: The role of diffusion-weighted imaging in patients with brain tumors. Am. J. Neuroradiol., 22 (6): 1081-8, 2001.

9- FAN G.G., DENG Q.L., WU Z.H., et al.: Usefulness of diffusion/perfusion weighted MRI in patients with nonenhancing supratentorial brain gliomas: A valuable tool to predict tumour grading? Br. J. Radiol., 79 (944): 6528, 2006.

10- YANG D., KOROGI Y., SUGAHARA T., et al.: Cerebral gliomas: Prospective comparison of multivoxel 2D chemical-shift imaging proton MR spectroscopy, echoplanar perfusion and diffusion-weighted MRI. Neuroradiology, 44: 656-66, 2002.

11- LAIPH, HO J.T., CHEN W.L., et al.: Brain abscess and necrotic brain tumor: Discrimination with proton MR spectroscopy and diffusion-weighted imaging. Am. J. Neuroradiol., 23 (8): 1369-77, 2002.

12-RIZZO S., GRECO P., GARCIA P., et al.: Role of diffusionand perfusion-weighted MR imaging for brain tumour characterization. Radiol. Med., 114: 645-59, 2009.

13- LAM W.W., POON W.S. and MATREWELI C.: Diffusion MR imaging in glioma: Does it have any role in the preoperation determination of grading of glioma? Clin. Radiol., 57: 219-25, 2002.

14-KNOPP M.V., GIESEL F.L., MARCOS H., et al.: Dynamic contrast-enhanced magnetic resonance imaging in oncology. Top. Magn. Reson. Imaging, 12: 301-8, 2001.

15- ELLIKA S.K., JAIN R., PATEL S.C., et al.: Role of Perfusion CT in Glioma Grading and Comparison with Conventional MR Imaging Features. American Journal of Neuroradiology, 28 (10): 1981-7, 2007.

16- SHIN J.H., LEE H.K., KWUN B.D., et al.: Using relative cerebral blood flow and volume to evaluate the histopathologic grade of cerebral gliomas: Preliminary results. Am. J. Roentgenol., 179: 783-9, 2002.

17- HAKYEMEZ B., ERDOGAN C., ERCAN I., et al.: Highgrade and low-grade gliomas: Differentiation by using perfusion. MR imaging Clin. Radiol., 60: 493-502, 2005.

18- LEV M.H., OZSUNAR Y., HENSON J.W., et al.: Glial tumor grading and outcome prediction using dynamic spin-echo MR susceptibility mapping compared with conventional contrast-enhanced MR: Confounding effect of elevated rCBV of oligodendrogliomas [corrected]. Am. J. Neuroradiol., 25: 214-21, 2004.

19- LAW M., YANG S., WANG H., et al.: Glioma gradings: Specificity and predictive values of perfusion MR imaging and proton MR spectroscopic imaging compared with conventional MR imaging. Am. J. Neuroradiol., 24: 198998, 2004.

20- ROLLIN N., GUYOTAT J., STREICHENBERGER N., et al.: Clinical relevance of diffusion and perfusion magnetic resonance imaging in assessing intra-axial brain tumors. Neuroradiology, 48: 150-9, 2006. 


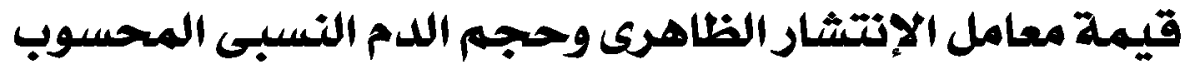

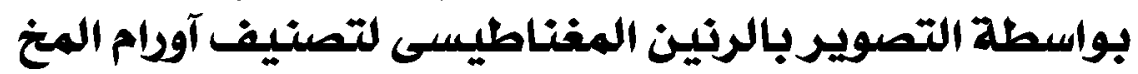

تعد آودام المـخ هي السبب الرئيسى الثاني للوفيات المرتبطة بالسرطان دون سن ال.ب وكذلك السبب الرنئيسى الثانى للوفيات المرتبطة

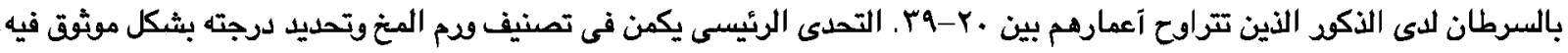

الهدف من الدراسة هو دراسة دود معامل الإنتشار الظاهرى وحجم الدم النسبى المحسوب بواسطة الرنين المغناطيسى لتصنيف آودام

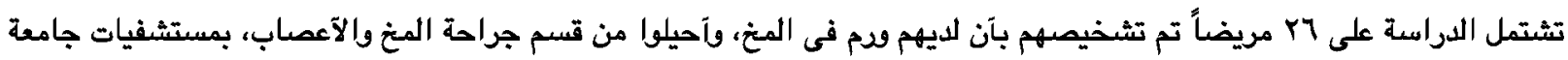

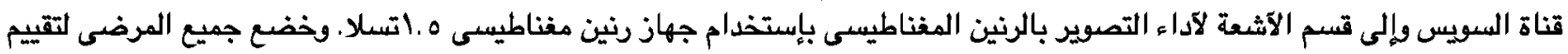

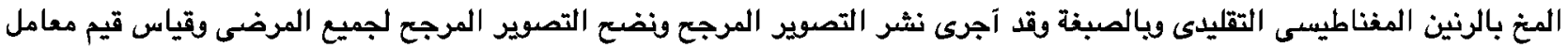

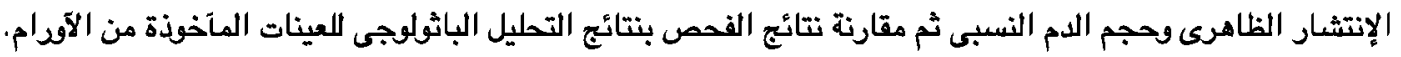

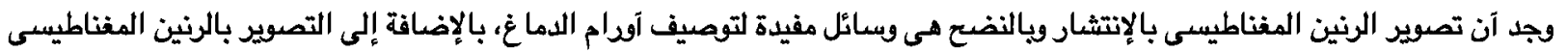

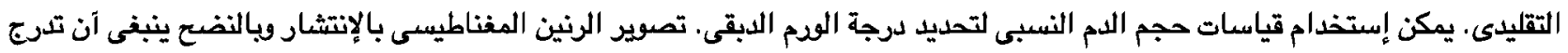

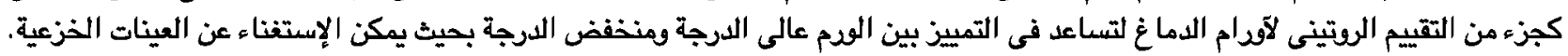
ومن الضرودى إجراء مزيد من البحوث لأتاكيد هذه النتائج. 